\title{
MS17-P07 | PhASE TRANSITIONS OF COPPER(I) IODIDE COMPOUNDS UNDER HIGH
}

\section{PRESSURE}

Gonzalez-Platas, Javier (Universidad de La Laguna, La Laguna, ESP); Aguirrechu-Comeron, Amagoia (Universidad de La Laguna, La Laguna, ESP); Rodriguez-Mendoza, Ulises R. (Universidad de La Laguna, La Laguna, ESP); Lavin della Ventura, Victor (Universidad de La Laguna, La Laguna, ESP); Conesa-Egea, Javier (Universidad Autonoma de Madrid, Madrid, ESP); Amo-Ochoa, Pilar (Universidad Autonoma de Madrid, Madrid, ESP)

Copper(I) iodide compounds can exhibit interesting mechanochromic and thermochromic luminescent properties with important technological applications. Mainly due to their large variety of structural configurations where Cul has the capability to form clusters with different dimensionality as rhomboid dimers, cubane, or staircase ladders [1-2]. It does a large variety of $\mathrm{Cu}$...Cu interactions giving attractive photoluminescence properties. Therefore, it could be interesting to explore new properties if we were able to control these interactions by applying external stimuli as temperature, grinding or pressure. We have done Xray diffraction and luminescence experiments under hihg pressure at room temperature and then we have performed the isothermal equation of state (EoS) for both compounds [3]. For $\mathrm{CuICl}_{2} \mathrm{Py}$ (1D staircase) we observe an isosymmetric phase transition of first order at 6GPa while for $(\mathrm{Cul})_{2}$ (Quin) (2D staircase) the phase transition is more subtle. It is a phase transition of second order around 3.5GPa. All these changes have been confirmed also in luminescence properties under pressure that we will show in the poster session.

[1] Q. Benito, I. Maurin, T. Cheisson, G. Nocton, A. Fargues, A. Garcia, C. Martineau, T. Gacoin, J.-P. Boilot, and S. Perruchas Chem. - A Eur. J., 2015, 21, pp. 5892-5897

[2] A. Aguirrechu-Comerón, R. Hernández-Molina, P. Rodríguez-Hernández, A. Muñoz, U. R. Rodríguez-Mendoza, V. Lavín, R. J. Angel, and J. Gonzalez-Platas, Inorg. Chem. 2016, 55, 7476-, vol. 55, no. 15, pp. 7476-7484.

[3] J. Gonzalez-Platas, M. Alvaro, F. Nestola, and R. Angel, J. Appl. Crystallogr., 2016, 49, 1377-1382. 